Commun. Fac. Sci. Univ. Ank. Ser. A1 Math. Stat.

Volume 69, Number 1, Pages 646-653(2020)

DOI: $10.31801 /$ cfsuasmas. 567734

ISSN 1303-5991 E-ISSN 2618-6470

http://communications.science.ankara.edu.tr/index.php?series=A1

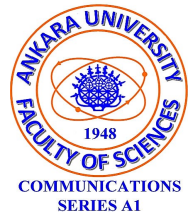

\title{
RESULTS ON QUASI-STATISTICAL LIMIT AND QUASI-STATISTICAL CLUSTER POINTS
}

\author{
İLKNUR ÖZGÜÇ
}

\begin{abstract}
In this paper we introduce the concepts of quasi-statistical limit point and quasi-statistical cluster point of a sequence. We give some inclusion results concerning these concepts. We also give the relationship between the Knopp core and quasi-statistical core of a sequence. Finally we state some theorems which deal with quasi-summability and quasi-statistical convergence of a sequence under some assumptions.
\end{abstract}

\section{INTRODUCTION}

The convergence of sequences has many generalizations with the aim of providing deeper insights into summability theory. One of the most important generalizations is statistical convergence [1, 2], 12, [14. It is quite effective especially when the classical limit does not exist since it is broader than ordinary convergence. Therefore concept of convergence has been studied by many authors [6], [7, [8], 9 , [13. It has also been used in number theory, trigonometric series and approximation theory [15], [16]. In [10] Ganichev and Kadets have introduced the concept of quasistatistical filter. Then by using the filter Özgüç and Yurdakadim have defined the quasi-statistical convergence and have studied the relationship between statistical convergence and quasi-statistical convergence in 11. The statistical analogues of limit points, limit superior, limit inferior and core of a sequence have been obtained by Fridy and Orhan [3], 4], 5].

In this study we introduce the concepts of quasi-statistical limit point and quasistatistical cluster point of a sequence. We give some inclusion results concerning these concepts. We also give the relationship between the Knopp core and quasistatistical core of a sequence. Finally we state some theorems which deal with quasi-summability and quasi-statistical convergence of a sequence under some assumptions.

Received by the editors: May 20, 2019, Accepted: November 26, 2019.

2010 Mathematics Subject Classification. 40A35, 40G15, 40F05.

Key words and phrases. Statistical convergence, quasi-statistical convergence, quasi-statistical limit points, quasi-statistical cluster points .

(C)2020 Ankara University Communications Faculty of Sciences University of Ankara-Series A1 Mathematics and Statistics 
Now let us recall the basic notations and definitions which we need throughout the paper.

If $K$ is a set of positive integers, $|K|$ will denote the cardinality of $K$. The natural density of $K$ is given by

$$
\delta(K)=\lim _{n \rightarrow \infty} \frac{1}{n}|\{k \leq n: k \in K\}|,
$$

if it exists.

The number sequence $x=\left(x_{k}\right)$ is statistically convergent to $L$ provided that for every $\varepsilon>0$ the set $K_{\varepsilon}=\left\{k \in \mathbb{N}:\left|x_{k}-L\right| \geq \varepsilon\right\}$ has natural density zero. In this case we write $s t-\lim x=L$.

Throughout the paper we assume that $c:=\left(c_{n}\right)$ is a sequence of positive real numbers such that

$$
\lim _{n} c_{n}=\infty \text { and } \limsup _{n} \frac{c_{n}}{n}<\infty .
$$

We define the quasi-density of $E \subset \mathbb{N}$ corresponding to the sequence $\left(c_{n}\right)$ by

$$
\delta_{c}(E):=\lim _{n} \frac{1}{c_{n}}|\{k \leq n: k \in E\}|
$$

if it exists.

The sequence $x=\left(x_{k}\right)$ is called quasi-statistically convergent to $L$ provided that for every $\varepsilon>0$ the set $E_{\varepsilon}=\left\{k \in \mathbb{N}:\left|x_{k}-L\right| \geq \varepsilon\right\}$ has quasi-density zero. In this case we write $s t_{q}-\lim x=L$ or $x_{k} \rightarrow L\left(s t_{q}\right)$.

The next result has been obtained in [11] to present the relationship between quasi-statistical convergence and statistical convergence.

Lemma 1. If $x=\left(x_{k}\right)$ is quasi-statistically convergent to $L$ then it is statistically convergent to $L$.

An example has been given in order to show that the converse of Lemma 1 does not hold (see [1]).

The following result has also been given to relate the statistical convergence and quasi-statistical convergence.

Under the assumptions (1) and

$$
d:=\inf _{n} \frac{c_{n}}{n}>0
$$

we immediately obtain that

" $x=\left(x_{k}\right)$ is statistically convergent to $L$ if and only if $x$ is quasi-statistically convergent to $L . "$

By $S_{q}$, we denote the set of all quasi-statistically convergent sequences.

It is easy to see that every convergent sequence is quasi-statistically convergent, i.e., $c \subset S_{q}$ where $c$ is the set of all convergent sequences.

If $x$ is a sequence we write $\left\{x_{k}: k \in \mathbb{N}\right\}$ to denote the range of $x$. If $\left\{x_{k(j)}: j \in \mathbb{N}\right\}$ is a subsequence of $x$ and $K=\{k(j): j \in \mathbb{N}\}$, then we abbreviate $\left\{x_{k(j)}\right\}$ by $\{x\}_{K}$. 
In case $\delta_{c}(K)=0,\{x\}_{K}$ is called a subsequence of quasi-density zero or a thin subsequence. On the other hand $\{x\}_{K}$ is called a nonthin subsequence of $x$ if $K$ does not have quasi density zero. Note that $\{x\}_{K}$ is a nonthin subsequence if either $\delta_{c}(K)$ is a positive number or does not exist.

The number $L$ is an ordinary limit point of a sequence $x$ if there is a subsequence of $x$ that converges to $L$.

Definition 2. The number $\lambda$ is a quasi-statistical limit point of the sequence $x$ if there is a nonthin subsequence which converges to $\lambda$.

Note that we will denote by $\Lambda_{x}^{c}, L_{x}$, the set of quasi-statistical limit points of $x$, and the set of ordinary limit points of $x$, respectively. It is clear that $\Lambda_{x}^{c} \subseteq L_{x}$ for any sequence $x$.

Proposition 3. $\Lambda_{x} \subseteq \Lambda_{x}^{c}$ holds where $\Lambda_{x}$ denotes the set of statistical limit points of $x$.

Proof. Let $\lambda \in \Lambda_{x}$. Then there exists a subset $M$ such that $\delta(M) \neq 0$ and $\{x\}_{M}$ converges to $\lambda$. One can write that

$$
\frac{1}{n}|\{k \leq n: k \in M\}| \leq H \frac{1}{c_{n}}|\{k \leq n: k \in M\}|
$$

where $H:=\sup _{n} \frac{c_{n}}{n}$ and it follows that $\delta_{c}(M) \neq 0$. This completes the proof.

It is known that under the conditions (1) and (2) quasi-statistical convergence coincides with statistical convergence. If we assume that the sequence $c=\left(c_{n}\right)$ satisfies the conditions (1) and (2), we have $\Lambda_{x}^{c}=\Lambda_{x}$.

Example 4. Define $x$ by

$$
x_{k}=\left\{\begin{array}{ccc}
r_{n} & ; & k=n^{2}, n=0,1,2, . . \\
k & ; & \text { otherwise }
\end{array}\right.
$$

where $\left\{r_{n}\right\}_{n=1}^{\infty}$ is a sequence whose range is the set of all rational numbers. It is known that $\Lambda_{x}=\varnothing, L_{x}=\mathbb{R}$ (see Example 2 of [3]). Since $\Lambda_{x}^{c} \subseteq \Lambda_{x}$, we get that $\Lambda_{x}^{c}=\varnothing$.

Definition 5. The number $\gamma$ is called a quasi-statistical cluster point of the sequence $x$ if the set $\left\{k \in \mathbb{N}:\left|x_{k}-\gamma\right|<\varepsilon\right\}$ does not have quasi-density zero for every $\varepsilon>0$.

We will denote the set of all quasi-statistical cluster points of $x$ by $\Gamma_{x}^{c}$. It is clear that $\Gamma_{x}^{c} \subseteq L_{x}$ for every sequence $x$.

Proposition 6. $\Gamma_{x} \subseteq \Gamma_{x}^{c}$ holds where $\Gamma_{x}$ denotes the set of all statistical cluster points of $x$. 
Proof. Let $\gamma \in \Gamma_{x}$. Then $\delta\left(M:=\left\{k \in \mathbb{N}:\left|x_{k}-\gamma\right|<\varepsilon\right\}\right) \neq 0$ for every $\varepsilon>0$. One can write that

$$
0 \neq \delta\left(\left\{k \in \mathbb{N}:\left|x_{k}-\gamma\right|<\varepsilon\right\}\right)=\frac{1}{n}|\{k \leq n: k \in M\}| \leq H \frac{1}{c_{n}}|\{k \leq n: k \in M\}|
$$

and it follows that $\delta_{c}(M) \neq 0$. This completes the proof.

Under the conditions (1) and (2), $\Gamma_{x}^{c}=\Gamma_{x}$ holds.

Following result presents the inclusion relationship between $\Gamma_{x}^{c}$ and $\Lambda_{x}^{c}$.

Theorem 7. For every sequence $x, \Lambda_{x}^{c} \subseteq \Gamma_{x}^{c}$ holds.

Proof. Let $\gamma \in \Lambda_{x}^{c}$. Then $\lim _{j} x_{k(j)}=\gamma$ and $\limsup _{n} \frac{1}{c_{n}}|\{k(j) \leq n\}|=r>0$ hold. Also the set $\left\{j:\left|x_{k(j)}-\gamma\right| \geq \varepsilon\right\}$ is finite for every $\varepsilon>0$ so

$$
\left\{k \in \mathbb{N}:\left|x_{k}-\gamma\right|<\varepsilon\right\} \supseteq\{k(j): j \in \mathbb{N}\} \text { - finite set. }
$$

Therefore

$$
\frac{1}{c_{n}}\left|\left\{k \leq n:\left|x_{k}-\gamma\right|<\varepsilon\right\}\right| \geq \frac{1}{c_{n}}|\{k(j): j \in \mathbb{N}\}|-\frac{1}{c_{n}} O(1) \geq \frac{r}{2}
$$

for infinitely many $n$. Hence $\delta_{c}\left(\left\{k \in \mathbb{N}:\left|x_{k}-\gamma\right|<\varepsilon\right\}\right) \neq 0$ for every $\varepsilon>0$ which completes the proof.

It is known that $\Lambda_{x}$ does not need to be closed but $\Gamma_{x}$ and $L_{x}$ are closed sets. In a similar proof to given by Fridy [3], one can also show the following.

Proposition 8. For any sequence $x$, the set $\Gamma_{x}^{c}$ is closed.

Theorem 9. If $\delta_{c}\left(\left\{k: x_{k} \neq y_{k}\right\}\right)=0$ then $\Lambda_{x}^{c}=\Lambda_{y}^{c}$ and $\Gamma_{x}^{c}=\Gamma_{y}^{c}$.

Proof. Assume that $\delta_{c}\left(\left\{k: x_{k} \neq y_{k}\right\}\right)=0$ and let $\lambda \in \Lambda_{x}^{c}$, the nonthin sequence $\{x\}_{K}$ converges to $\lambda$. Note that $\delta_{c}\left(\left\{k: x_{k}=y_{k}\right\}\right) \neq 0$. Therefore the latter set yields a nonthin subsequence $\{y\}_{K^{\prime}}$ of $\{y\}_{K}$ which converges to $\lambda$. Hence $\Lambda_{x}^{c} \subseteq \Lambda_{y}^{c}$. By symmetry one can also get $\Lambda_{x}^{c} \supseteq \Lambda_{y}^{c}$. The second assertion can be proved in a similar way.

The following theorem is easy to prove by using the same technique in Theorem 2 of [3]. Therefore we omit it.

Theorem 10. If $x$ is a number sequence then there exists a sequence $y$ such that $L_{y}=\Gamma_{x}^{c}$ and $\delta_{c}\left(\left\{k: x_{k} \neq y_{k}\right\}\right)=0$. Moreover, the range of $y$ is a subset of the range of $x$.

Another noteworthy and useful result concerning the quasi-statistical cluster points is as follows.

Theorem 11. If $x$ is a number sequence that has a bounded nonthin subsequence, then $x$ has a quasi-statistical cluster point. 
Proof. For such $x$, the above theorem ensures that there exists a sequence $y$ such that $L_{y}=\Gamma_{x}^{c}$ and $\delta_{c}\left(\left\{k: x_{k} \neq y_{k}\right\}\right)=0$. Then $y$ must have a bounded nonthin subsequence, so by the Bolzano-Weierstrass Theorem $L_{y} \neq \varnothing$ which implies $\Gamma_{x}^{c} \neq$ $\varnothing$.

Now we immediately get the following corollary.

Corollary 12. If $x$ is a bounded sequence, then $x$ has a quasi-statistical cluster point.

Theorem 13. If $x$ is a bounded sequence then it has a thin subsequence $\{x\}_{K}$ such that $\left\{x_{k}: k \in \mathbb{N}-K\right\} \cup \Gamma_{x}^{c}$ is a compact set.

Proof. Again by the above results one can choose a bounded sequence $y$ such that $L_{y}=\Gamma_{x}^{c},\left\{y_{k}: k \in \mathbb{N}\right\} \subseteq\left\{x_{k}: k \in \mathbb{N}\right\}$, and $\delta_{c}(K)=0$ where $K:=\left\{k \in \mathbb{N}: x_{k} \neq y_{k}\right\}$. This implies

$$
\left\{x_{k}: k \in \mathbb{N}-K\right\} \cup \Gamma_{x}^{c}=\left\{y_{k}: k \in \mathbb{N}\right\} \cup L_{y}
$$

and the right-hand set is compact.

\section{Quasi-Statistical Limit Superior and Inferior}

The aim of this section is to present quasi-statistical limit superior and inferior to obtain some quasi-statistical analogues of ordinary limit superior, inferior and statistical limit superior, inferior as in [4. We also introduce quasi-statistical core of a sequence and prove some results.

Definition 14. If $x$ is a real number sequence then the quasi-statistical limit superior and inferior of $x$ are defined by

$$
\begin{aligned}
& s t_{q}-\limsup x=\left\{\begin{array}{cl}
\sup B_{x}^{c} & , \text { if } B_{x}^{c} \neq \emptyset \\
-\infty & , \text { if } B_{x}^{c}=\emptyset
\end{array},\right. \\
& s t_{q}-\liminf x=\left\{\begin{array}{cl}
\inf A_{x}^{c} & , \text { if } A_{x}^{c} \neq \emptyset \\
+\infty, & \text { if } A_{x}^{c}=\emptyset
\end{array}\right.
\end{aligned}
$$

where $B_{x}^{c}=\left\{b \in \mathbb{R}: \delta_{c}\left(\left\{k: x_{k}>b\right\}\right) \neq 0\right\}, A_{x}^{c}=\left\{a \in \mathbb{R}: \delta_{c}\left(\left\{k: x_{k}<a\right\}\right) \neq 0\right\}$. We now give a simple example to understand the concepts just defined.

Example 15. Let $c:=\left(c_{n}\right)$ be the sequence of positive real numbers such that $\lim _{n} c_{n}=\infty$, and $\lim _{n} \frac{\sqrt{n}}{c_{n}}=\infty$. We can choose a subsequence $\left\{c_{n_{p}}\right\}$ such that $c_{n_{p}}>1$ for each $p \in \mathbb{N}$.

Consider the sequence $x=\left(x_{k}\right)$ defined by

$$
x_{k}:=\left\{\begin{array}{cc}
c_{k}, \quad k \text { is square and } c_{k} \in\left\{c_{n_{p}}: p \in \mathbb{N}\right\} \\
2, \quad k \text { is square and } c_{k} \notin\left\{c_{n_{p}}: p \in \mathbb{N}\right\} \\
1, \quad k \text { is odd and } k \text { is not square } \\
0, \quad k \text { is even and } k \text { is not square }
\end{array} .\right.
$$

One can easily see that $s t_{q}-\lim \sup x=1, s t_{q}-\liminf x=0$. 
Definition 16. The real number sequence $x$ is said to be quasi-statistically bounded if there is a number $M$ such that $\delta_{c}\left(\left\{k \in \mathbb{N}:\left|x_{k}\right|>M\right\}\right)=0$.

The sequence $x$ in the above example is not quasi-statistically convergent but quasi-statistically bounded. Also note that quasi-statistical boundedness implies that $s t_{q}-\lim \sup , s t_{q}-\lim \inf$ are finite and if the sequence is quasi-statistically bounded then $s t_{q}-\lim \sup x$ is the greatest element of the set of quasi-statistical cluster points and $s t_{q}-\lim \inf x$ is the least element of this set.

Now we investigate the relationship between $s t_{q}-\lim \sup x$ and $s t-\lim \sup x$ and also the relationship between $s t_{q}-\lim \inf x$ and $s t-\lim \inf x$.

Remark 17. Let $H<\infty$. Then

$$
s t_{q}-\liminf x \leq s t-\liminf x \leq s t-\lim \sup x \leq s t_{q}-\lim \sup x
$$

holds for any real sequence.

Proof. Let $\alpha_{2}=s t_{q}-\liminf x$ and $\alpha_{1}=s t-\liminf x$. Then $\delta\left(\left\{k: x_{k}<\alpha_{1}+\varepsilon\right\}\right) \neq$ 0 and $\delta\left(\left\{k: x_{k}<\alpha_{1}-\varepsilon\right\}\right)=0$ holds for every $\varepsilon>0$. Since $H<\infty$, we have that $\delta\left(\left\{k: x_{k}<\alpha_{1}+\varepsilon\right\}\right) \leq \delta_{c}\left(\left\{k: x_{k}<\alpha_{1}+\varepsilon\right\}\right)$ and this implies $\delta_{c}\left(\left\{k: x_{k}<\alpha_{1}+\varepsilon\right\}\right) \neq$ 0 . Then

$$
\alpha_{1}+\varepsilon \in A_{x}^{c}=\left\{a \in \mathbb{R}: \delta_{c}\left(\left\{k: x_{k}<a\right\}\right) \neq 0\right\}
$$

and it is known that $\inf A_{x}^{c}=\alpha_{2}$ which implies $\alpha_{2} \leq \alpha_{1}+\varepsilon$ for every $\varepsilon>0$. $\varepsilon$ is arbitrary and we obtain that $\alpha_{1}=s t-\liminf x \geq \alpha_{2}=s t_{q}-\liminf x$. Now let $\beta_{2}=s t_{q}-\lim \sup x, \beta_{1}=s t-\lim \sup x$. Then $\delta\left(\left\{k: x_{k}>\beta_{1}-\varepsilon\right\}\right) \neq 0$ and $\delta\left(\left\{k: x_{k}>\beta_{1}+\varepsilon\right\}\right)=0$ holds for every $\varepsilon>0$. Since $H<\infty$ we have that $\delta\left(\left\{k: x_{k}>\beta_{1}-\varepsilon\right\}\right) \leq \delta_{c}\left(\left\{k: x_{k}>\beta_{1}-\varepsilon\right\}\right)$ and this implies $\delta_{c}\left(\left\{k: x_{k}>\beta_{1}-\varepsilon\right\}\right) \neq$ 0 . Then

$$
\beta_{1}-\varepsilon \in B_{x}^{c}=\left\{b \in \mathbb{R}: \delta\left(\left\{k: x_{k}>b\right\}\right) \neq 0\right\}
$$

and it is known that $\sup B_{x}^{c}=\beta_{2}$ which implies $\beta_{1}-\varepsilon \leq \beta_{2}$ for every $\varepsilon>0$. $\varepsilon$ is arbitrary and we obtain that $\beta_{1}=s t-\lim \sup x \leq \beta_{2}=s t_{q}-\lim \sup x$ which completes the proof.

Knopp has introduced the concept of the core of a sequence and has proved the well known core theorem. In order to produce natural analogues of Knopp core and statistical core of a sequence, we can replace limit points and statistical cluster points with quasi-statistical cluster points in [4, [5].

Definition 18. If $x$ is a quasi-statistically bounded real sequence then the quasistatistical core of $x$ is the closed interval st $t_{q}-\operatorname{core}\{x\}=\left[s t_{q}-\liminf x, s t_{q}-\lim \sup x\right]$. In case $x$ is not quasi-statistically bounded, st $t_{q}$-core $\{x\}$ is defined accordingly as either $\left[s t_{q}-\lim \inf x, \infty\right),(-\infty, \infty)$ or $\left(-\infty, s t_{q}-\lim \sup x\right]$.

One can easily see from Remark 1 that

$$
\text { st }-\operatorname{core}\{x\} \subseteq s t_{q}-\operatorname{core}\{x\} \subseteq K-\operatorname{core}\{x\} .
$$


Recall that the sequence $x=\left(x_{k}\right)$ is said to be strongly quasi-summable to $L$ if

$$
\lim _{n} \frac{1}{c_{n}} \sum_{k=1}^{n}\left|x_{k}-L\right|=0 \text {. }
$$

The space of all strongly quasi-summable sequences is denoted by $N_{q}$.

$$
N_{q}:=\left\{x \text { : for some } L, \lim _{n} \frac{1}{c_{n}} \sum_{k=1}^{n}\left|x_{k}-L\right|=0\right\} .
$$

Also the sequence $x=\left(x_{k}\right)$ is said to be quasi-summable to $L$ if

$$
\lim _{n} \frac{1}{c_{n}} \sum_{k=1}^{n} x_{k}=L
$$

Now we can give a result concerning with the quasi-summability and quasistatistical limit superior.

Theorem 19. Let the sequence $x$ is bounded above, $\ell=\delta_{c}(\mathbb{N})$ and $\beta l=s t_{q}-$ $\lim \sup x$. If the sequence $x$ is quasi-summable to $\beta \ell^{2}$ then $x$ is quasi-statistically convergent to $\beta \ell$.

Proof. Suppose that $x$ is not quasi-statistically convergent to $\beta \ell$. Then $s t_{q}-\lim \inf x<$ $\beta l$, therefore there is a number $\mu<\beta l$ such that $\delta_{c}\left(\left\{k: x_{k}<\mu\right\}\right) \neq 0$. Let $K^{\prime}:=\left\{k: x_{k}<\mu\right\}$. Then by the definition of quasi-statistical limit superior, $\delta_{c}\left(\left\{k: x_{k}>\beta l+\varepsilon\right\}\right)=0$, for every $\varepsilon>0$. Now define

$$
K^{\prime \prime}:=\left\{k: \mu \leq x_{k} \leq \beta l+\varepsilon\right\}, K^{\prime \prime \prime}:=\left\{k: x_{k}>\beta l+\varepsilon\right\}, B:=\sup x<\infty .
$$

Since $\delta_{c}\left(K^{\prime}\right) \neq 0$, there are infinitely many $n$ such that

$$
\frac{1}{c_{n}}\left|K_{n}^{\prime}\right| \geq r>0
$$

for each such $n$ we have

$$
\begin{aligned}
\frac{1}{c_{n}} \sum_{k=1}^{n} x_{k} & =\frac{1}{c_{n}} \sum_{k=1, k \in K_{n}^{\prime}}^{n} x_{k}+\frac{1}{c_{n}} \sum_{k=1, k \in K_{n}^{\prime \prime}}^{n} x_{k}+\frac{1}{c_{n}} \sum_{k=1, k \in K_{n}^{\prime \prime \prime}}^{n} x_{k} \\
& <\frac{\mu}{c_{n}}\left|K_{n}^{\prime}\right|+\frac{\beta \ell+\varepsilon}{c_{n}}\left|K_{n}^{\prime \prime}\right|+\frac{B}{c_{n}}\left|K_{n}^{\prime \prime \prime}\right| \\
& =\frac{\mu}{c_{n}}\left|K_{n}^{\prime}\right|+(\beta \ell+\varepsilon)\left(\ell-\frac{\left|K_{n}^{\prime}\right|}{c_{n}}\right)+o(1) \\
& \leq(\mu-\beta \ell) \frac{\left|K_{n}^{\prime}\right|}{c_{n}}+\beta \ell^{2}+\varepsilon\left(\ell-\frac{\left|K_{n}^{\prime}\right|}{c_{n}}\right) \\
& \leq \beta \ell^{2}-(\beta \ell-\mu) \frac{\left|K_{n}^{\prime}\right|}{c_{n}}+\varepsilon(\ell-r)+o(1)
\end{aligned}
$$




$$
\leq \beta \ell^{2}-(\beta \ell-\mu) r+\varepsilon(\ell-r)+o(1)
$$

Since $\varepsilon$ is arbitrary it follows that $\liminf \frac{1}{c_{n}} \sum_{k=1}^{n} x_{k}<\beta \ell^{2}$. Hence $x$ is not quasisummable to $\beta \ell^{2}$ and this completes the proof.

This theorem is a generalization of Theorem 5 in [3]. Using the symmetry, we also have the following for lower bounds.

Corollary 20. Let the sequence $x$ is bounded below, $\ell=\delta_{c}(\mathbb{N})$ and $\alpha l=s t_{q}-$ $\lim \inf x$. If the sequence $x$ is quasi-summable to $\alpha \ell^{2}$ then $x$ is quasi-statistically convergent to $\alpha \ell$.

\section{REFERENCES}

[1] Fast, H., Sur la convergence statistique, Colloq. Math., 2 (1951), 241-244.

[2] Fridy, J. A., On statistical convergence, Analysis, 5 (1985) 301-313.

[3] Fridy, J. A., Statistical limit points, Proc. Amer. Math. Soc., 118 (1993), 1187-1192.

[4] Fridy, J. A. and Orhan,C., Statistical limit superior and limit inferior, Proc. Amer. Math. Soc., 125 (1997), 3625-3631.

[5] Fridy, J. A. and Orhan, C., Statistical core theorems, J. Math. Anal. Appl., 208 (1997), $520-527$.

[6] Connor, J., The statistical and strong p-Cesàro convergence of sequences, Analysis, 8 (1988) 47-63.

[7] Connor, J., On strong matrix summability with respect to a modulus and statistical convergence, Canad. Math. Bull., 32 (1989), 194-198.

[8] Connor, J., Demirci, K. and Orhan, C., Multipliers and factorization for bounded statistically convergence sequences, Analysis (Munich) 22(4) (2002), 321-333.

[9] Demirci, K. and Orhan, C., Bounded multipliers of bounded A-statistically convergent sequences, Journal of Mathematical Analysis and Applications, 235 (1999), 122-129.

[10] Ganichev, M. and Kadets, V., Filter convergence in Banach spaces and generalized bases, Taras Banach (Ed.), General Topology in Banach Spaces, NOVA Science Publishers, Huntington, New York (2001), 61-69.

[11] Sakaoğlu Özgüç, I. and Yurdakadim, T., On quasi-statistical convergence, Commun. Fac. Sci. Univ. Ank. Series A1, 61 (2012), 11-17.

[12] Šalát, T., On statistically convergent sequences of real numbers, Math. Slovaca, 30(2) (1980), 139-150.

[13] Schoenberg, I. J., The integrability of certain functions and related summability methods, Amer. Math. Monthly, 66 (1959), 361-375.

[14] Steinhaus, H., Sur la convergence ordinarie et la convergence asymtotique, Colloq. Math., 2 (1951), 73-74.

[15] Yurdakadim, T., Taş, E. and Sakaoğlu, İ., Approximation of functions by the sequence of integral operators, Appl. Math. Comput., 219 (2012), 3863-3871.

[16] Zygmund, A., Trigonometric series, 2nd Ed. Cambridge Univ. Press, 1979.

Current address: İlknur Özgüç: Ankara University, Department of Mathematics, Ankara, Turkey

E-mail address: i.ozguc@gmail.com

ORCID Address: http://orcid.org/0000-0002-6622-8249 